\title{
Insights into Ultrafast Demagnetization in Pseudogap Half-Metals
}

\author{
Andreas Mann, ${ }^{*}$ Jakob Walowski, and Markus Münzenberg ${ }^{\dagger}$ \\ I. Physikalisches Institut, Georg-August-Universität Göttingen, Göttingen, Germany \\ Stefan Maat, Matthew J. Carey, and Jeffrey R. Childress \\ San Jose Research Center, HGST, San Jose, California 95135, USA \\ Claudia Mewes \\ Department of Physics and Astronomy, University of Alabama, Tuscaloosa, Alabama 35487, USA \\ Daniel Ebke, Volker Drewello, Günter Reiss, and Andy Thomas \\ Department of Physics, Universität Bielefeld, Bielefeld, Germany \\ (Received 28 February 2012; published 15 November 2012)
}

\begin{abstract}
Interest in femtosecond demagnetization dynamics was sparked by Bigot's experiment in 1996, which unveiled the elementary mechanisms that relate the electrons' temperature to their spin order. Simultaneously, the application of fast demagnetization experiments has been demonstrated to provide key insight into technologically important systems such as high-spin-polarization metals, and consequently there is broad interest in further understanding the physics of these phenomena. To gain new and relevant insights, we performed ultrafast optical pump-probe experiments to characterize the demagnetization processes of highly spin-polarized magnetic thin films on a femtosecond time scale. Full spin polarization is obtained in half-metallic ferro- or ferrimagnets, where only one spin channel is populated at the Fermi level, whereas the other one exhibits a gap. In these materials, the spin-scattering processes is controlled via the electronic structure, and thus their ultrafast demagnetization is solely related to the spin polarization via a Fermi golden-rule model. Accordingly, a long demagnetization time correlates with a high spin polarization due to the suppression of the spin-flip scattering at around the Fermi level. Here we show that isoelectronic Heusler compounds $\left(\mathrm{Co}_{2} \mathrm{MnSi}, \mathrm{Co}_{2} \mathrm{MnGe}\right.$, and $\left.\mathrm{Co}_{2} \mathrm{FeAl}\right)$ exhibit a degree of spin polarization between $59 \%$ and $86 \%$. We explain this behavior by considering the robustness of the gap against structural disorder. Moreover, we observe that $\mathrm{CoFe}$-based pseudogap materials, such as partially ordered $\mathrm{Co}-\mathrm{Fe}-\mathrm{Ge}$ and $\mathrm{Co}-\mathrm{Fe}-\mathrm{B}$ alloys, can reach similar values of the spin polarization. By using the unique features of these metals we vary the number of possible spin-flip channels, which allows us to pinpoint and control the half-metals' electronic structure and its influence on the elementary mechanisms of ultrafast demagnetization.
\end{abstract}

DOI: 10.1103/PhysRevX.2.041008

Subject Areas: Condensed Matter Physics, Materials Science, Spintronics

Since the discovery of ultrafast demagnetization processes on femtosecond time scales, the underlying mechanism has been under debate [1,2]. However, the last few years have seen the development of the first quantitative models, such as the microscopic three-temperature model [3], the stochastic Landau-Lifshitz-Bloch equation describing averaged spin ensembles [4,5], and stochastic atomistic descriptions [6]. These models suggest that the spin-scattering $\tau_{\mathrm{el}-\mathrm{sp}}$ is related to the Gilbert damping

\footnotetext{
*Now at Laboratory for Ultrafast Microscopy and Electron Scattering, École Polytechnique Fédérale de Lausanne (EPFL), 1015 Lausanne, Switzerland.

Corresponding author mmuenze@gwdg.de

Published by the American Physical Society under the terms of the Creative Commons Attribution 3.0 License. Further distribution of this work must maintain attribution to the author(s) and the published article's title, journal citation, and DOI.
}

parameter $\alpha$ that describes the energy dissipation of the magnetic system in quasiequilibrium via the same elementary spin-flip processes [7]. The Gilbert damping tends to be small in half-metals where the elementary spin-flip processes are blocked [8]. Just recently, progress in the ab initio description of Gilbert damping has been made [9], shedding additional light onto a long-standing issue. By correlating the experimentally observed values of the Gilbert damping parameter $\alpha$ to the coupling parameter of the magnetic system (magnons) and the electron temperature, the Landau-Lifshitz-Bloch model allows the quantitative description of ultrafast demagnetization versus time without any free parameters [10]. The relationship between the Gilbert damping $\alpha$, the spin scattering at equilibrium, and its connection to ultrafast processes has been summarized in a recent review [11]. Nevertheless, alternative theoretical model calculations have recently reopened the discussion of the underlying mechanisms of ultrafast demagnetization and suggest [12-14] ballistic, 
diffusive, or superdiffusive spin currents to be the contributing or even dominating mechanisms. Moreover, coherent effects have been discussed to influence ultrafast magnetization dynamics on the shortest time scales [15], as they are especially important in insulating materials. Here, we present investigations of the spin-dependent electronic structure, in particular, the half-metallic gap size and its relation to the ultrafast energy relaxation. A half-metallic gap of $300 \mathrm{meV}$ in one spin channel allows for the suppression of spin-flip processes by a factor of 40 compared to the non-spin-polarized case, but only in a small energy window of less than $150 \mathrm{meV}$. A similar suppression of the spin-flip scattering can be achieved in pseudogap materials with nonperfect half-metallicity over a larger energy window. By engineering the spin-dependent electronic structure of the materials, this allows us to control the ultrafast dynamics by a factor of 2 to 4 . In the following, we will describe how we control the elementary spin-flip processes by design in our experiments.

In a half-metal, only one spin type is present at the Fermi level [16,17]. Consequently, the electron-related properties have the highest possible spin-dependent asymmetry. This property makes these materials interesting for applications in the field of spin electronics [18] and more recently spin caloritronics [19,20]. Several different classes of halfmetallic materials have been investigated: Magnetic oxides such as $\mathrm{CrO}_{2}, \mathrm{La}_{0.66} \mathrm{Sr}_{0.33} \mathrm{MnO}_{3}$, and $\mathrm{Fe}_{3} \mathrm{O}_{4}$ exhibit halfmetallic behavior. However, even though $\mathrm{CrO}_{2}$ has been shown to have a perfect half-metallic electronic structure, it has been difficult to utilize this material in tunneling magnetoresistance (TMR) devices due to its challenging preparation [21]. Much better suited for experiments, with the possibility of gradual control of the spin polarization, are Heusler compounds, which represent a promising and versatile class of materials. Some ferromagnetic Heusler compounds are half-metallic, others are found to be superconducting [22], and recently, some have been suggested to belong to the novel class of topological insulators [23]. In the Heusler structure, it is possible to achieve high spinpolarization by combining different elements to shift the Fermi energy level into the middle of the band gap. This can be accomplished by substituting one of the constituents with an element of a different electron count, i.e., to add or to remove electrons [24]. There has been steady progress in using Heusler materials in magnetic tunnel junctions. So far, up to $220 \%$ magnetoresistance has been observed at room temperature [25]. However, a strong temperature dependence in these Heusler-based tunnel junctions is generally observed, and their TMR ratio typically drops by a factor of 2 between low and room temperature; the mechanism responsible for this behavior is not clear to date [26]. In addition, half-metallic Heusler compounds ( $L 2_{1}$ phase) are very sensitive to structural disorder, giving rise to the formation of defect states deep in the band gap [27] that lead to a reduction of the half-metallic gap.
Crystallization in the fully ordered $L 2_{1}$ phase is necessary to achieve maximum spin-polarization, but this typically requires high-temperature preparation methods. However, the partially ordered $B 2$ phase can be achieved at lower preparation temperatures and for some Heusler compounds also exhibits relatively high spin polarization. By using this wide array of Heusler compounds, we can deliberately tune the degree of spin polarization in a sample, e.g., by opening and reducing the number of spin-flip relaxation channels.

Ultrafast pump-probe experiments measure the demagnetization dynamics on a femtosecond time scale [2]. Electrons are photoexcited by a laser pulse $(1.5 \mathrm{eV})$. Efficient thermalization by spin-flip and non-spin-flip electron-electron scattering in the first 10 fs leads to a fast decay of highly energetic electrons that can be observed as a fast, steplike demagnetization. For a half metal, however, the blocking of spin-flip scattering processes starts to be efficient once all the electrons are relaxed below the energy of the half-metallic gap. Then, the electron system and the spin system are basically isolated, and the spin-flip scattering probability is reduced to zero because no states are available in one of the spin channels. Since the electronic system and the spin system are thermally decoupled, the demagnetization time is determined by the weak spin-lattice interaction. Using a Fermi golden rule to model the elementary spin-flip processes, transition rates at the Fermi level are found to depend on the spin polarization $P$ via a prefactor of $(1-P)$ [8]. It is evident from the assumptions that this model is only valid for gaps larger than the thermal energy. The typical time scale for this process is found to be greater than $100 \mathrm{ps}$ for the oxide half metals.

For half-metallic Heusler compounds with high spin polarization, so far no evidence of the expected spin-flip blocking has been identified [8,28], opening up questions on the current understanding of the underlying mechanisms of spin-flip processes, ultrafast demagnetization, and how the properties of the Heusler compound are related to the spin-dependent electronic structure? Previously, it was shown that the magnetization of Heusler compounds follows a Slater-Pauling curve [29]. Thus, it is reasonable to apply a rigid-band picture, where the Fermi level can be shifted simply by changing the number of valence electrons. With that assumption, in a recent study, the ultrafast spin dynamics of $\mathrm{Co}_{2} \mathrm{MnSi}$ and $\mathrm{Co}_{2} \mathrm{FeSi}$ Heusler compounds were investigated by femtosecond laser excitation. In these materials, the Fermi level is located close to the bottom of the gap for $\mathrm{Co}_{2} \mathrm{MnSi}$ and close to the top of the gap for $\mathrm{Co}_{2} \mathrm{FeSi}$. Consequently, states of both bands in $\mathrm{Co}_{2} \mathrm{MnSi}$ and $\mathrm{Co}_{2} \mathrm{FeSi}$ become thermally populated, even for small excitation energies. These states are not completely blocked from providing a channel for fast demagnetization. Simulations reveal that the electron and spin dynamics behave in a semiconductorlike manner: Excitation and relaxation channels can be traced in selected bands. For the $\mathrm{Co}_{2} \mathrm{MnSi}\left(\mathrm{Co}_{2} \mathrm{FeSi}\right)$ 
case, a channel of hot holes below (above) the gap dominates the relaxation. In another study, the blocking of spin-flip processes in a series of $\mathrm{Co}_{2} \mathrm{MnAl}_{x} \mathrm{Si}_{1-x}$ and $\mathrm{Co}_{2} \mathrm{Fe}_{x} \mathrm{Mn}_{1-x} \mathrm{Si}$ compounds [30,31] was identified by measuring the Gilbert damping parameter using ferromagnetic resonance. In that study, the Fermi level is shifted from the bottom of the gap for 28 electrons $\left(\mathrm{Co}_{2} \mathrm{MnAl}\right)$ and to the top of the gap for 30 electrons $\left(\mathrm{Co}_{2} \mathrm{FeSi}\right)$. A strong dependence of the Gilbert damping with a minimum at $\mathrm{Co}_{2} \mathrm{Fe}_{0.4} \mathrm{Mn}_{0.6} \mathrm{Si}$ (29.4 electrons) was found. Concurrently, a band-structure calculation shows that the total density of states at the Fermi level decreases to a minimum. The damping increases as the Fermi level enters the conduction band, and the half-metallic character breaks down.

In our study, we do not vary the number of electrons, but rather focus on comparing spin-flip processes for the isoelectronic compounds, $\mathrm{Co}_{2} \mathrm{MnSi}, \mathrm{Co}_{2} \mathrm{MnGe}$, and $\mathrm{Co}_{2} \mathrm{FeAl}$, which all have 29 contributing electrons. The Fermi level should thus be unchanged and located in the gap region. The half-metallic film's growth parameters were optimized using feedback from previous spinpolarized transport experiments. For the optimized growth conditions, $\mathrm{Co}_{2} \mathrm{FeAl}$ layers were directly grown onto $\mathrm{MgO}$, followed by a $\mathrm{MgO}$ tunnel barrier and a $\mathrm{CoFe}$ counterelectrode with known spin polarization as a reference. The $\mathrm{CO}_{2} \mathrm{FeAl}$ layers showed a pronounced $B 2$ order that increased with annealing temperature [Fig. 1(a)]. The integrated intensity of the (400) peak gradually increases, indicating the increase of the quality of the sample with temperature. In the femtosecond demagnetization experiments, the time-resolved Kerr rotation is measured for $\mathrm{Co}_{2} \mathrm{FeAl}$ films that were annealed at various temperatures [Fig. 1(b)]. The pump-probe measurements were carried out using a double-modulated time-resolved magnetooptic Kerr effect setup [32], and at the same time, timeresolved reflectivity of the samples was measured using balanced photodiodes. As the annealing temperature is increased, we observe the demagnetization time $\tau_{m}$ to increase from 350(5) to 385(5) fs simultaneously with the integrated (400) peak intensity and thus with the $B 2$ order. This time is significantly slower compared to the case of $\mathrm{Ni}$. For this $\mathrm{Co}_{2} \mathrm{FeAl}$ film, the value of the spin polarization extracted from the TMR measurements with a $\mathrm{Co}_{70} \mathrm{Fe}_{30}$ counterelectrode was $86 \%$ [Fig. 1(c)] [33], compared to $45 \%$ for Ni $[34,35]$. From the Fermi golden-rule model, which gives $\tau_{\text {el-sp }} \sim(1-P)^{-1}$, a slower demagnetization time below the picosecond time range, is expected, matching the observed trend.

In the following study, several materials are compared: three isoelectronic Heusler compounds $\left(\mathrm{Co}_{2} \mathrm{MnSi}\right.$, $\mathrm{Co}_{2} \mathrm{FeAl}$, and $\mathrm{Co}_{2} \mathrm{MnGe}$ ), two Co-Fe-based materials (Co-Fe-Ge and $\mathrm{Co}-\mathrm{Fe}-\mathrm{B}$ ), and the half-Heusler compound $\mathrm{CoMnSb}$, which is also expected to develop half-metallic features, together with standard $\mathrm{Ni}, \mathrm{Ni}_{81} \mathrm{Fe}_{19}, \mathrm{CoFe}$, and $\mathrm{CrO}_{2}$. The demagnetization curves (Fig. 2) are normalized to their demagnetization values at the maximum and shifted for clarity. While Ni's demagnetization is given as a reference at the bottom, the figure shows that the position of the maximum demagnetization $\tau_{\max }$ of $\mathrm{Co}_{2} \mathrm{FeAl}$ increases to about 1 ps. No steplike signature-a specific contribution of nonequilibrium electrons to the demagnetization (e.g., for less than $100 \mathrm{fs}$ ) — is found. The shift of the maximum demagnetization is small compared to our earlier work on oxide half-metals, where a maximum of 84 ps was obtained for $\mathrm{CrO}_{2}$ [8]. Here, the previous approach to compare values of $\tau_{\max }$ is extended to consider small differences in the polarization that lead to small differences (less than $10 \mathrm{fs}$ ) in $\tau_{\max }$. We extract the value of the demagnetization time scale $\tau_{m}$ using rate equations based on the three-temperature model by Beaurepaire and co-workers [1]. The coupling strengths between the three temperature reservoirs determine the demagnetization time scale $\tau_{m}$. Our interpretation is based on an analytical solution of this three-temperature model using the sum of
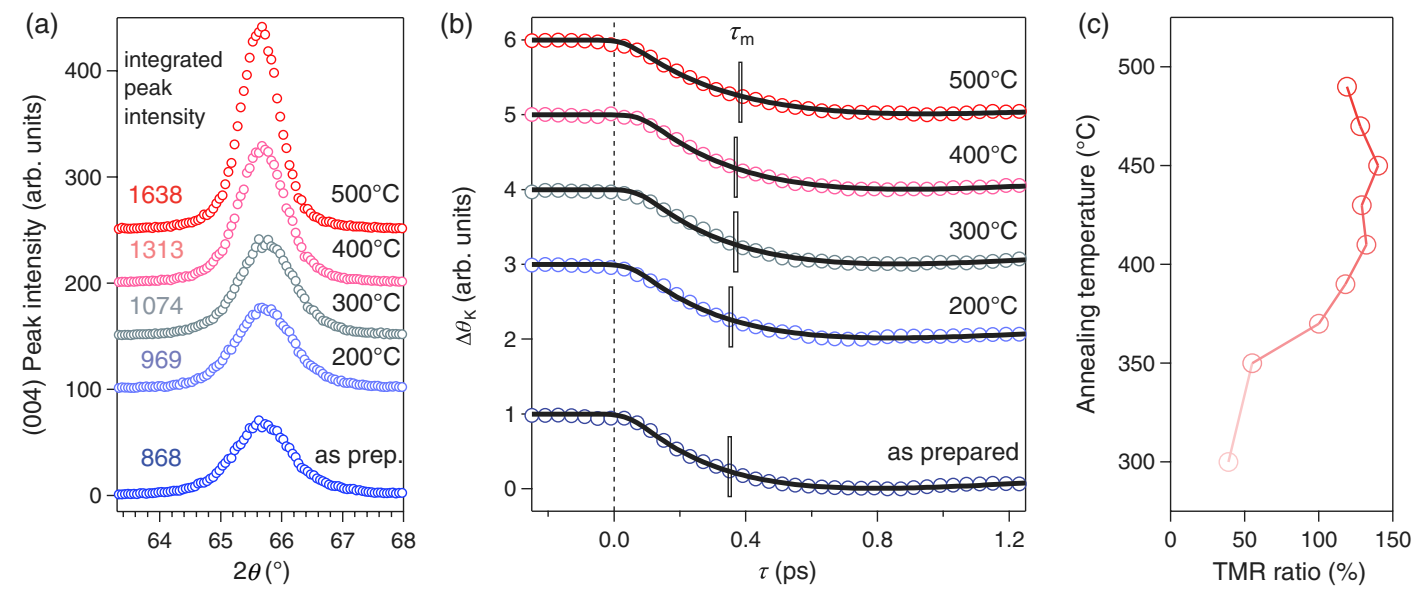

FIG. 1. Film optimization. Annealing temperature dependence of $\mathrm{Co}_{2} \mathrm{FeAl}$. Panel (a) depicts the x-ray diffraction 004-peak intensity, (b) shows the demagnetization time (vertical bar marks the value of $\tau_{m}$ ), the lines are fits using an analytical function derived from a three-temperature model, and (c) exhibits the $\mathrm{TMR}$ of the $\mathrm{Co}_{2} \mathrm{FeAl} / \mathrm{MgO} / \mathrm{CoFe}$ junctions. 


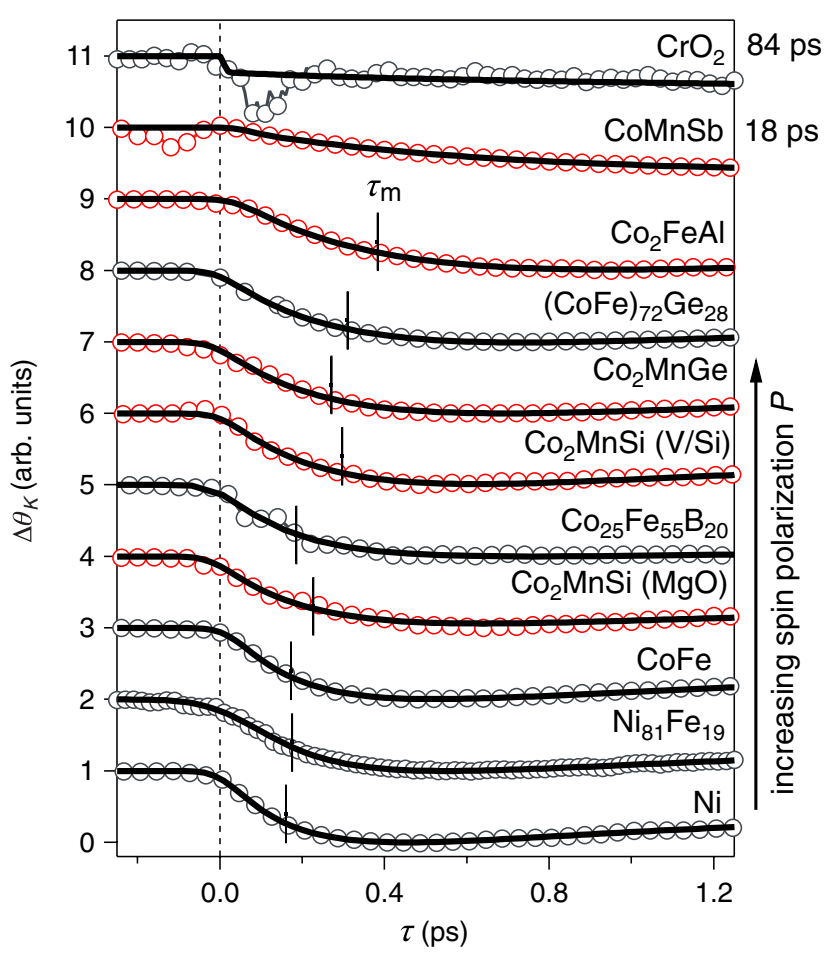

FIG. 2. Ultrafast demagnetization spectra. Femtosecond pump-probe experiments for different materials are sorted by their spin polarization $P$. Heusler compounds are indicated by red data points. The lines are fits using an analytical function derived from a three-temperature model, and the vertical bar marks the value of $\tau_{m}$.

two exponential functions [36], related to the two time scales: $\tau_{m}$ and the electron-phonon scattering $\tau_{\text {el-lat }}$. It should be noted that, while we do not consider diffusion processes in the model because we can disregard them in the first few picoseconds, we must fit four parameters (two time scales and two amplitudes). However, one of the parameters, the electron-phonon scattering time $\tau_{\text {el-lat }}$, can be fixed by using reflectivity measurements [37].

In Fig. 3, the demagnetization time $\tau_{m}$ is plotted against the spin-polarization values of the materials. The values of $\tau_{m}$ range from $160 \mathrm{fs}(\mathrm{Ni})$ to $380 \mathrm{fs}\left(\mathrm{Co}_{2} \mathrm{FeAl}\right)$, while the spin polarization ranges from $45 \%(\mathrm{Ni})$ to $86 \%\left(\mathrm{Co}_{2} \mathrm{FeAl}\right)$. Notably, the isoelectronic Heusler compounds $\mathrm{Co}_{2} \mathrm{MnSi}$, $\mathrm{Co}_{2} \mathrm{FeAl}$, and $\mathrm{Co}_{2} \mathrm{MnGe}$ exhibit spin polarizations $P$ in the range of $59 \%$ to $86 \%$. The values for $P$ for each material are derived from their spin-transport properties: Ni [34,35], CoFe [38], Co-Fe-B [39], $\mathrm{Co}_{2} \mathrm{MnSi}$ on Si [40], $(\mathrm{CoFe})_{0.72} \mathrm{Ge}_{0.28}$ [41], $\mathrm{Co}_{2} \mathrm{MnGe}$ [42], $\mathrm{Co}_{2} \mathrm{FeAl}$ [43], $\mathrm{CoMnSb}$ (theo.) [44], and $\mathrm{CrO}_{2}$ [45] (see table and data in the Supplemental Material [46]). These results fill the gap of intermediate polarization values between standard ferromagnetic metals and near-100\% spin-polarized oxides and display the connection between demagnetization times $\tau_{m}$ and spin polarization $P$ given by the golden-rule model, shown as lines in Fig. 3. For low spin polarization, the demagnetization time scale $\tau_{m}$ is dominated by the fast electron-spin relaxation rate $\tau_{m} \approx \tau_{\text {el-sp }}$ [8]. The intersection of the ordinate for $P=0$ is determined by the average momentum-scattering rate $\tau_{\mathrm{el}, 0} / c^{2}=100 \mathrm{fs}$, where $c$ is the spin-orbit-coupling mixing parameter. Different values for the anisotropy relaxation rate $\tau_{\text {lat-sp }}$ determine the limiting values at $P=1$ as $\tau_{m} \approx \tau_{\text {lat-sp }}=3 \mathrm{ps}$ and $1 \mathrm{~ns}$ (the lower red line and upper black line in Fig. 3), and are the only free parameters of the model. The demagnetization time scale $\tau_{m}$ follows the expected evolution indicating the suppression of the spin-flip scattering $\tau_{\mathrm{el}-\mathrm{sp}} \sim$ $(1-P)^{-1}$ as the dominant factor.

To confirm our results that $\mathrm{Co}_{2} \mathrm{FeAl}$ has the highest spin polarization among the full Heusler compounds investigated, we collected additional electronic structure data of the half-metallic films using tunneling spectroscopy. The conductance curves $(d I / d V$ versus bias voltage) presented in Fig. 4(a), show the signature of a half-metallic gap of $300 \mathrm{meV}$ for $\mathrm{Co}_{2} \mathrm{FeAl}$, which is absent in the case of $\mathrm{Co}_{2} \mathrm{MnSi}$. This gap is also evident in the suppression of the inelastic magnon peak at negative bias voltages [Fig. 4(b)]. Both indicate a half-metallic nature for this material. Therefore, one would also naively expect a much larger demagnetization time. If we take $\tau_{\mathrm{el}-\mathrm{sp}} \sim$ $(1-P)^{-1}$ as the dominating factor in the simple Fermi golden-rule model from Müller et al. [8], one can estimate the demagnetization time to increase by a factor of 4 from $170 \mathrm{fs}$ as measured for $P=0.45(\mathrm{Ni})$ to about $700 \mathrm{fs}$ for $P=0.86\left(\mathrm{Co}_{2} \mathrm{FeAl}\right)$, which is higher but in reasonable agreement with our observed value of $380 \mathrm{fs}$ for $\mathrm{Co}_{2} \mathrm{FeAl}$ (Fig. 3). To further improve the model and get more insight into the contributing spin-flip mechanisms, thermal effects have to be considered as the relatively small half-metallic gap of $300 \mathrm{meV}$ observed in $\mathrm{Co}_{2} \mathrm{FeAl}$ must be taken into account.

In Fig. 3, we used a one-band model that disregarded the electron temperature. This model is also justified in a multiband situation if the density of states can be described with one averaged polarization value and if the halfmetallic gap is larger than the thermal energy of the electrons. In particular, this is the case if the gap is large and the Fermi level is located in the half-metallic gap and is separated from the band edges by an energy larger than $k_{B} T$. Then, Fermi's golden-rule approach for the density of states at the Fermi level can be applied. The spin-mixing parameter $c$ describes the spin-flip transitions at the Fermi level. In a band structure with spin-orbit interaction, intermixing of spin-up and spin-down states leads to a spin flip with some probability if the electron is scattered into a hot spot [47], characterized by transition matrix elements (the spin-orbit-coupling mixing parameter is given by $c=\frac{\zeta_{\text {so }}}{\Delta E_{\text {ex }}}$ $[8,11,48]$, i.e., the ratio of spin-orbit coupling energy and exchange energy). Spin mixing was first addressed by Elliott [45], who related spin-flip transitions to spin-orbit coupling. The golden-rule approach does not necessarily 


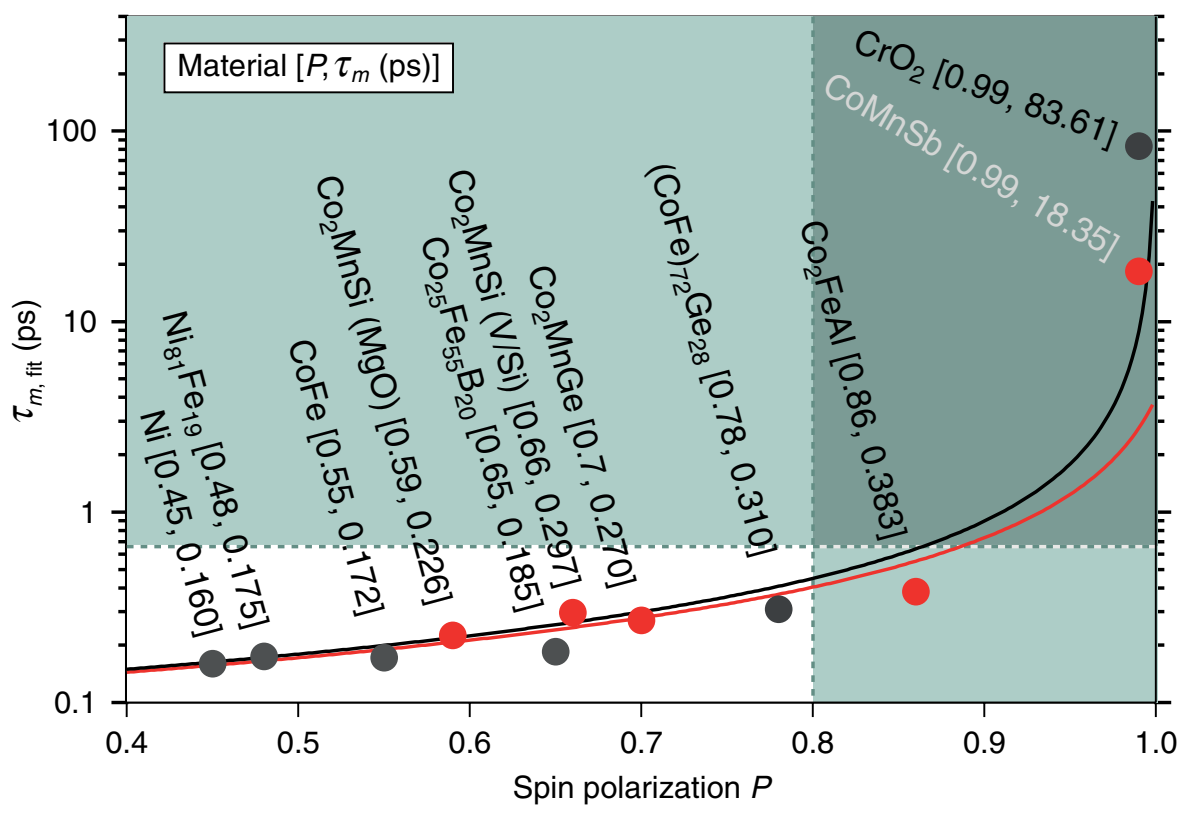

FIG. 3. Demagnetization time $\tau_{m}$ versus spin polarization $P$. The half-metallic properties can be classified in a $P$ versus $\tau_{m}$ plot. If the points lie on top of the lines given, spin-flip blocking is the dominant mechanism describing the simultaneous increase of $P$ and $\tau_{m}$. The lines are model calculations using Fermi's golden-rule approach showing the $\tau_{\mathrm{el}-\mathrm{sp}} \sim(1-P)^{-1}$ behavior. For each material the demagnetization time $\tau_{m}$ taken from Fig. 2 is plotted as a function of the spin polarization $P$. Note the logarithmic scale for $\tau_{m}$. Intersections are $P=0, \tau_{m}=\frac{\tau_{\mathrm{el}, 0}}{c^{2}}=100 \mathrm{fs}$ and $P=1, \tau_{m}=3 \mathrm{ps}$ or $1 \mathrm{~ns}$, respectively. Heusler compounds are given in red. In brackets the value of $P$ and $\tau_{m}$ (fs) is given.

make any prediction concerning the mechanism of the spin-flip scattering and thus $c$ could originate from any non-spin-conserving process with similar strength replacing the transition probability or microscopic mechanism related to asymmetry of the spin-dependent density of states for high spin polarization in general. However, the spin-mixing mechanism has been successfully applied for the recent $a b$ initio description of the Gilbert damping, which is, at the moment, the only approach describing its temperature-dependent features [9]. Despite these
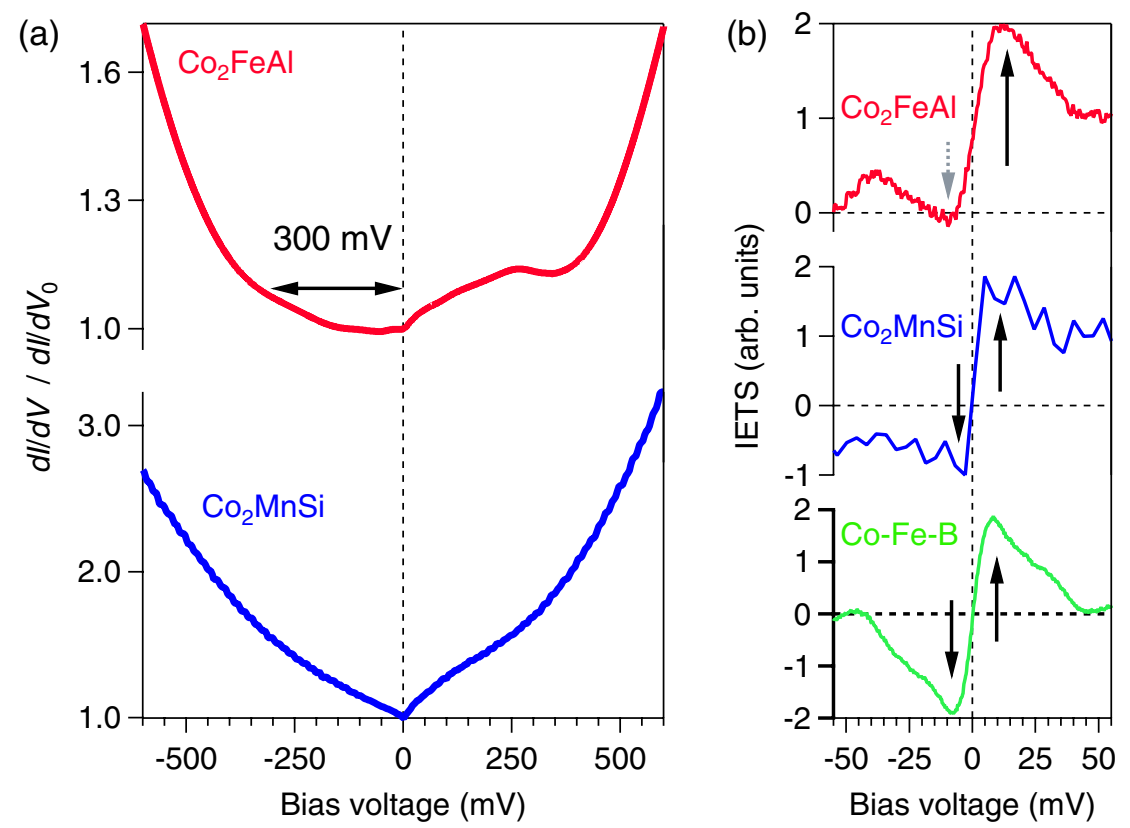

FIG. 4. Probing the electronic structure. (a) Tunneling spectroscopy of Heusler-based magnetic tunnel junctions. A gaplike feature of $300 \mathrm{mV}$ is visible in the $\mathrm{Co}_{2} \mathrm{FeAl}$ spectrum. (b) Inelastic electron-tunneling spectra of the Heusler-based and the reference Co-Fe-B junctions. In $\mathrm{Co}_{2} \mathrm{FeAl}$, the magnon peak at negative bias is suppressed (compare Ref. [42]). 

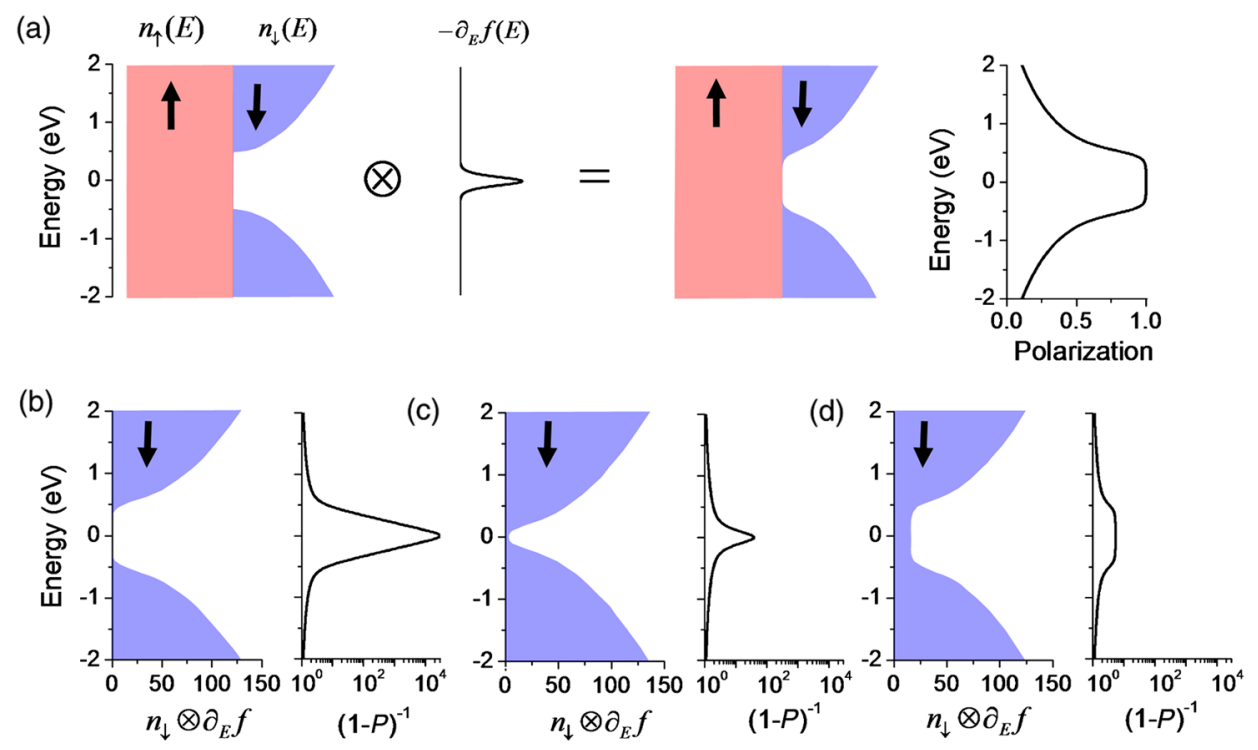

FIG. 5. Energy dependent golden-rule model. (a) Model density of states for a one-spin channel convoluted with temperaturedependent kernel with a width corresponding to $T=600 \mathrm{~K}$ (approximately $60 \mathrm{meV}$ ) given by the derivative of the Fermi distribution $-\partial_{E} f(E)$, followed by the according spin polarization $P$. In panels (b)-(d) the influence of the thermal excitations on the spin-flip suppression factor $\tau_{\mathrm{el}-\mathrm{sp}} \sim(1-P)^{-1}$ is calculated in analogy to (a) for three cases: a large gap of $1 \mathrm{eV}$ (b), a small gap of $300 \mathrm{meV}$ (experimental value for $\mathrm{Co}_{2} \mathrm{FeAl}$ ) in the spin down density of states (c), as well as for a pseudogap, representing the case of Co-FeGe (d). The suppression factor is given on a logarithmic scale.

simplifications, the golden-rule model has been successful in most cases even though all parameters have so far been treated as being band and energy independent.

A temperature kernel, given by the derivative of the Fermi function (temperatures studied were $T=$ $300,600 \mathrm{~K}$ ), is used to describe the effect of temperature on the band structure, which is depicted in Fig. 5. After a convolution of the density of states with the temperature kernel, the 300-mV-wide gap of $\mathrm{Co}_{2} \mathrm{FeAl}$ as determined by tunneling spectroscopy is significantly blurred due to the thermal excitations. This demonstrates that for these smallgap materials the model needs to be extended for thermal effects. To describe the temperature dependence of the magnetic Gilbert damping, Elliott's approach has been extended to a band-resolved method by Kamberský [49]. By averaging all possible transitions over the Brillouin zone, including thermal occupation functions, he derived a spin-scattering rate where the temperature-dependent kernel functions are given by $k_{\mu, \nu}$ for band index $\mu$ and $\nu$, and $\left|\phi_{\mu, \nu}\right|^{2}$ is the transition probability;

$$
\begin{aligned}
\tau_{\mathrm{el}-\mathrm{sp}}^{-1} & =\left\langle\sum_{\mu, \nu}\left|\phi_{\mu, \nu}\right|^{2} k_{\mu, \nu} \delta\left(E_{\mu}-E_{\nu}-\omega\right)\right\rangle_{\mathrm{el}, \mathrm{lat}} \\
& =\frac{c^{2}}{\tau_{\mathrm{el}, 0}}\left\langle\sum_{\mu, \nu}\left[1-P(E)_{\mu, \nu}\right]\left[-\partial_{E} f_{\mu}(E)\right] \delta\left(E_{\mu}-E_{\nu}\right)\right\rangle \text { el, lat }
\end{aligned}
$$

In Eq. (2), we formulate the transition probability in terms of the band's polarization $P_{\mu, \nu}$ and spin-mixing parameter $c$ (see details in the Supplemental Material [46]). The temperature-dependent kernels $k_{\mu, \nu}$ give rise to intraband (same index) transitions dominating at low temperatures and interband (different index) transitions with spin flips at higher temperatures, whose effect on the ultrafast demagnetization was also discussed by Fähnle in [50] and later reevaluated by Carva [51], explicitly taking into account the electron-phonon and nonequilibrium contributions. The effect is illustrated in Fig. 5 for several specific examples. For $\mu=\nu$ and taking into account only thermal smearing by the electron distribution, the temperature kernel function is given by the derivative of the Fermi distribution $k_{\mu}=-\partial_{E} f(E)$, which is shown in Fig. 5(a) for $T=600 \mathrm{~K}$. The resulting thermally broadened density of states is then calculated by a convolution, and subsequently, the spin polarization $P(E)$ is obtained as explicitly shown for the $1-\mathrm{eV}$ case in Fig. 5(a). Three schematic band structures, one with 1-eV-wide gap, one with a 300-meV-wide gap, and the third with a pseudogap where the density of states is reduced to one-fifth in one spin channel (equal to a moderate spin polarization of $P=$ 0.67 ) over a $1-\mathrm{eV}$-wide gap, are discussed in the following. To compare these three cases, the factor $(1-P)^{-1}$ is calculated in Figs. 5(b)-5(d), which is the relevant factor suppressing the spin-flip scattering $\tau_{\text {el-sp }}$. Clearly, for materials like $\mathrm{Co}_{2} \mathrm{FeAl}$ that exhibit a relatively small band gap, the effect of thermal smearing cannot be disregarded. The momentum-scattering time $\tau_{\mathrm{el}, 0}$ is defined by the total number of scattering channels in momentum space (e.g., 
in an insulator, this reduces to zero). At a temperature of $600 \mathrm{~K}$, the suppression factor for spin scattering is $10^{4}$ in a 1-eV gap, it decreases to 40 for a small gap of $300 \mathrm{meV}$ in a narrow energy window of less than $150 \mathrm{meV}$, and it falls off to 10 for a $100-m e V$ gap (not shown). By contrast, for the pseudogap case, a comparable suppression of a factor of 5 is found in a wide energy window of $1.1 \mathrm{eV}$. To derive a model relating the spin-relaxation rate to $P$ at the Fermi level and the half-metallic gap size, we assume an average scattering rate $\tau_{\mathrm{el}, 0}$ and band-independent polarization $P$. Using the approximations for the Fermi distribution's tail, the expression can be rewritten using the Boltzmann occupation for the electrons and holes, yielding

$\tau_{\mathrm{el}-\mathrm{sp}} \approx \frac{\tau_{\mathrm{el}, 0}}{c^{2}}\left[1-\left(P\left(E_{F}\right)-\frac{\left(2 m^{*}\right)^{3 / 2}}{(2 \pi)^{2} \hbar^{3}}\left(k_{B} T\right)^{3 / 2} e^{-E_{\mathrm{gap}} / k_{B} T}\right)\right]^{-1}$.

Here the polarization $P$ and, accordingly, the factor $(1-P)^{-1}$ is decreased by a Boltzmann probability term that accounts for the thermal excitations. This equation yields quantitative numbers in the simple model limit for one band, taking into account the reduced spin-flip processes in small-band-gap materials. We can conclude that, for Heusler compounds, a gradual transition of the spin-flip probability can be controlled by increasing the half metallicity (gap width), which is consistent with our observations. On the other hand, for systems with a small band gap of $100 \mathrm{meV}$, thermal effects from the heated electron system lead to a very strong reduction of the spin-flip blocking.

The only exception is CoMnSb, which shows the slowest demagnetization time of $18 \mathrm{ps}$. However, the sample does not display a high-spin-polarization value as determined by spin-transport experiments. In this case, an additional effect becomes dominant in determining $\tau_{m}$. This effect originates from the material's low Curie temperature of only $474 \mathrm{~K}$, which leads to a thermally induced slowdown of the magnetization's decay. This can be demonstrated using either the Landau-Lifshitz-Bloch equation [10] or the microscopic three-temperature model [3]. It is a signature of magnetic fluctuations and excited spin waves [52] becoming even more dominant for high spin polarizations, so-called type-II materials $[3,53]$. In a fluence-dependent experiment, such a behavior was identified and separated from the typical behavior of a half metal.

How can we understand the present results? The largest spin polarization in the isoelectronic row of Heusler compounds is found for $\mathrm{Co}_{2} \mathrm{FeAl}$, which is striking because it is not expected by band-structure calculations. However, among the materials investigated $\mathrm{Co}_{2} \mathrm{Fe} A l$ exhibits the best $B 2$ order after annealing at rather low temperature. This is reflected in an increase of the TMR ratio and explains the unexpected superior half-metallic behavior and the increase in the demagnetization time to $380 \mathrm{fs}$, a manifestation of the spin-flip blocking on ultrafast time scales. For Co-Fe-Ge, no perfect half-metallic behavior is predicted, but rather a pseudogap around the Fermi level is exhibited [54,55]. In pseudogap materials, the same mechanisms as in the Heusler compounds are acting to increase the spin polarization. Adding an $s p$ atom to the two transition metal's $d$ states with mixed valence (here the ferromagnets $\mathrm{Co}$ and $\mathrm{Fe}$ ) changes the electronic structure via specific bonds [44]; thus alloying of $\mathrm{CoFe}$ with $\mathrm{Ge}$ generates gaplike features in the density of states without requiring perfect long-range structural order. In $B 2$-based alloys, this is realized, however, on a local scale, so that the large spin polarization is robust against disorder in the alloy films and consequently a large increase in the demagnetization time to $310 \mathrm{fs}$ is found. Similarly, these strategies can be applied to suppress spin-flip processes at the Fermi level for applications requiring a low magnetic damping or high spin polarization that is robust against structural disorder. Combinations of materials with Co-Fe- $X$, where the third element $X$ is from group 13 (boron group) to 16 (chalcogenides) [39,56-58], open up new possibilities for designing electronic states and magnetic properties [59]. In such pseudogap materials, the large spin polarization is obtained by a locally induced partial suppression of states in one spin channel. Simultaneously, it is possible to block spin-flip processes and thus to suppress spin scattering on ultrafast time scales. Models adding temperature effects allow a qualitative prediction of the spin-flip suppression for small-gap half-metals.

In summary, we have shown slow demagnetization in femtosecond pump-probe experiments for a series of thinfilm materials with a wide range of spin polarization. Under optimized growth conditions for the materials investigated, the demagnetization time can be increased from 100 fs (for $P=0 \%$ ) to approximately 380 fs (for $P=$ $86 \%)$. The increase can be understood by the factor of $(1-P)^{-1}$ derived from a golden-rule approach without considering thermal effects. Considering the gap size and thermal smearing, we can also describe this increase more quantitatively. Our experiments allow us to pinpoint one important mechanism for ultrafast demagnetization directly related to the spin-dependent electronic structure.

A.T. and V.D. acknowledge the MIWF of the NRW state government for financial support. M. M. and J.W. acknowledge the funding provided by the German research foundation (DFG) via the SFB 602. A. T., M. M., and G. R. acknowledge the funding provided by the German research foundation (DFG) via the SpinCaT priority program SPP 1538.

[1] E. Beaurepaire, J. C. Merle, A. Daunois, and J. Y. Bigot, Ultrafast Spin Dynamics in Ferromagnetic Nickel, Phys. Rev. Lett. 76, 4250 (1996).

[2] A. Kirilyuk, A. Kimel, and T. Rasing, Ultrafast Optical Manipulation of Magnetic Order, Rev. Mod. Phys. 82, 2731 (2010). 
[3] B. Koopmans, G. Malinowski, F. Dalla Longa, D. Steiauf, M. Fähnle, T. Roth, M. Cinchetti, and M. Aeschlimann, Explaining the Paradoxical Diversity of Ultrafast LaserInduced Demagnetization, Nature Mater. 9, 259 (2009).

[4] N. Kazantseva, U. Nowak, and R. Chantrell, Slow Recovery of the Magnetisation after a Sub-Picosecond Heat Pulse, Europhys. Lett. 81, 27004 (2008).

[5] U. Atxitia, O. Chubykalo-Fesenko, N. Kazantseva, D. Hinzke, U. Nowak, and R. W. Chantrell, Micromagnetic Modeling of Laser-Induced Magnetization Dynamics Using the Landau-Lifshitz-Bloch Equation, Appl. Phys. Lett. 91, 232507 (2007).

[6] I. Radu, K. Vahaplar, C. Stamm, T. Kachel, N. Pontius, H. A. Dürr, T. A. Ostler, J. Barker, R. F. L. Evans, R. W. Chantrell et al., Transient Ferromagnetic-Like State Mediating Ultrafast Reversal of Antiferromagnetically Coupled Spins, Nature (London) 472, 205 (2011).

[7] U. Atxitia and O. Chubykalo-Fesenko, Ultrafast Magnetization Dynamics Rates Within the LandauLifshitz-Bloch Model, Phys. Rev. B 84, 144414 (2011).

[8] G. M. Müller, J. Walowski, M. Djordjevic, G.-X. Miao, A. Gupta, A. V. Ramos, K. Gehrke, V. Moshnyaga, K. Samwer, J. Schmalhorst et al., Spin Polarization in HalfMetals Probed by Femtosecond Spin Excitation, Nature Mater. 8, 56 (2009).

[9] K. Gilmore, Y. U. Idzerda, and M. D. Stiles, Identification of the Dominant Precession-Damping Mechanism in Fe, Co, and Ni by First-Principles Calculations, Phys. Rev. Lett. 99, 027204 (2007); A. Brataas, Y. Tserkovnyak, and G. E. W. Bauer, Scattering Theory of Gilbert Damping, Phys. Rev. Lett. 101, 037207 (2008); H. Ebert, S. Mankovsky, D. Ködderitzsch, and P. Kelly, $A b$ initio Calculation of the Gilbert Damping Parameter via the Linear Response Formalism, Phys. Rev. Lett. 107, 066603 (2011).

[10] U. Atxitia and O. Chubykalo-Fesenko, Evidence for Thermal Mechanisms in Laser-Induced Femtosecond Spin Dynamics, Phys. Rev. B 81, 174401 (2010).

[11] See review M. Fähnle and C. Illg, Electron Theory of Fast and Ultrafast Dissipative Magnetization Dynamics, J. Phys. Condens. Matter 23, 493201 (2011).

[12] M. Battiato, K. Carva, and P. Oppeneer, Superdiffusive Spin Transport as a Mechanism of Ultrafast Demagnetization, Phys. Rev. Lett. 105, 027203 (2010).

[13] A. Melnikov, I. Razdolski, T. Wehling, E. Papaioannou, V. Roddatis, P. Fumagalli, O. Aktsipetrov, A. Lichtenstein, and U. Bovensiepen, Ultrafast Transport of Laser-Excited Spin-Polarized Carriers in $\mathrm{Au} / \mathrm{Fe} / \mathrm{MgO}(001)$, Phys. Rev. Lett. 107, 076601 (2011); M. Wietstruk, A. Melnikov, C. Stamm, T. Kachel, N. Pontius, M. Sultan, C. Gahl, M. Weinelt, H. Dürr and U. Bovensiepen, Hot-ElectronDriven Enhancement of Spin-Lattice Coupling in Gd and $\mathrm{Tb}$ if Ferromagnets Observed by Femtosecond X-Ray Magnetic Circular Dichroism, Phys. Rev. Lett. 106, 127401 (2011).

[14] K. Carva, M. Battiato, and P. Oppeneer, Ab initio Investigation of the Elliott-Yafet Electron-Phonon Mechanism in Laser-Induced Ultrafast Demagnetization, Phys. Rev. Lett. 107, 207201 (2011); S. Essert and H. Schneider, Electron-Phonon Scattering Dynamics in Ferromagnetic Metals and their Influence on Ultrafast
Demagnetization Processes, Phys. Rev. B 84, 224405 (2011).

[15] L. Guidoni, E. Beaurepaire, and J.-Y. Bigot, Magnetooptics in the Ultrafast Regime: Thermalization of Spin Populations in Ferromagnetic Films, Phys. Rev. Lett. 89, 017401 (2002); J.-Y. Bigot, M. Vomir, and E. Beaurepaire, Coherent Ultrafast Magnetism Induced by Femtosecond Laser Pulses, Nature Phys. 5, 515 (2009); H. Vonesch and J.-Y. Bigot, arXiv:1201.0166v1.

[16] R. de Groot, F. Mueller, P. Engen, and K. Buschow, New Class of Materials: Half-Metallic Ferromagnets, Phys. Rev. Lett. 50, 2024 (1983).

[17] See review by M. Ventkatesan, in Novel Materials, edited by H. Kronmüller and S.S.P. Parkin, Handbook of Magnetism and Advanced Magnetic Materials Vol. 4 (John Wiley and Sons, New York, 2007).

[18] G.-X. Miao, M. Münzenberg, and J.S. Moodera, Tunneling Path toward Spintronics, Rep. Prog. Phys. 74, 036501 (2011).

[19] G.E.W. Bauer, E. Saitoh, and B.J. van Wees, Spin Caloritronics, Nature Mater. 11, 391 (2012); G. E.W. Bauer, A.H. MacDonald, and S. Maekawa, Spin Caloritronics, Solid State Commun. 150, 459 (2010).

[20] M. Walter, J. Walowski, V. Zbarsky, M. Münzenberg, M. Schäfers, D. Ebke, G. Reiss, A. Thomas, P. Peretzki, M. Seibt et al., Seebeck Effect in Magnetic Tunnel Junctions, Nature Mater. 10, 742 (2011).

[21] G.-X. Miao and A. Gupta, in Nanoscale Magnetic Materials and Applications, edited by J.P. Liu, E. Fullerton, O. Gutfleisch, and D. J. Sellmyer (Springer, New York, 2009).

[22] B. Balke, G. Fecher, J. Winterlik, and C. Felser, MnGa, a Compensated Ferrimagnet with High Curie Temperature and Low Magnetic Moment for Spin Torque Applications, Appl. Phys. Lett. 90, 152504 (2007).

[23] S. Chadov, X. Qi, J. Kuebler, G. H. Fecher, C. Felser, and S.C. Zhang, Tunable Multifunctional Topological Insulators in Ternary Heusler Compounds, Nature Mater. 9, 541 (2010).

[24] T. Graf, C. Felser, and S. S. P. Parkin, Simple Rules for the Understanding of Heusler Compounds, Prog. Solid State Chem. 39, 1 (2011).

[25] S. Tsunegi, Y. Sakuraba, M. Oogane, K. Takanashi, and Y. Ando, Large Tunnel Magnetoresistance in Magnetic Tunnel Junctions Using a $\mathrm{Co}_{2} \mathrm{MnSi}$ Heusler Alloy Electrode and a MgO Barrier, Appl. Phys. Lett. 93, 112506 (2008).

[26] L. Chioncel, Y. Sakuraba, E. Arrigoni, M. Katsnelson, M. Oogane, Y. Ando, T. Miyazaki, E. Burzo, and A. Lichtenstein, Nonquasiparticle States in $\mathrm{Co}_{2} \mathrm{MnSi}$ Evidenced through Magnetic Tunnel Junction Spectroscopy Measurements, Phys. Rev. Lett. 100, 086402 (2008).

[27] S. Picozzi, A. Continenza, and A. Freeman, Role of Structural Defects on the Half-Metallic Character of $\mathrm{Co}_{2} \mathrm{MnGe}$ and $\mathrm{Co}_{2} \mathrm{MnSi}$ Heusler Alloys, Phys. Rev. B 69, 094423 (2004).

[28] D. Steil, S. Alebrand, T. Roth, M. Krauß, T. Kubota, M. Oogane, Y. Ando, H. Schneider, M. Aeschlimann, and M. Cinchetti, Band-Structure-Dependent Demagnetization in the Heusler Alloy $\mathrm{Co}_{2} \mathrm{Mn}_{1-x} \mathrm{Fe}_{x} \mathrm{Si}$, Phys. Rev. Lett. 105, 217202 (2010). 
[29] H. C. Kandpal, G. H. Fecher, and C. Felser, Calculated Electronic and Magnetic Properties of the Half-Metallic, Transition Metal Based Heusler Compounds, J. Phys. D 40, 1507 (2007).

[30] M. Oogane, T. Kubota, Y. Kota, S. Mizukami, H. Naganuma, A. Sakuma, and Y. Ando, Gilbert Magnetic Damping Constant of Epitaxially Grown Co-Based Heusler Alloy Thin Films, Appl. Phys. Lett. 96, 252501 (2010).

[31] T. Kubota, S. Tsunegi, M. Oogane, S. Mizukami, T. Miyazaki, H. Naganuma, and Y. Ando, Half-Metallicity and Gilbert Damping Constant in $\mathrm{Co}_{2} \mathrm{Fe}_{x} \mathrm{Mn}_{1-x} \mathrm{Si}$ Heusler Alloys Depending on the Film Composition, Appl. Phys. Lett. 94, 122504 (2009).

[32] M. Djordjevic, G. Eilers, A. Parge, M. Münzenberg, and J.S. Moodera, Intrinsic and Nonlocal Gilbert Damping Parameter in all Optical Pump-Probe Experiments, J. Appl. Phys. 99, 08F308 (2006).

[33] D. Ebke, P. Thomas, O. Schebaum, M. Schaefers, D. Nissen, V. Drewello, A. Huetten, and A. Thomas, Low B2 Crystallization Temperature and High Tunnel Magnetoresistance in $\mathrm{Co}_{2} \mathrm{FeAl} / \mathrm{MgO} / \mathrm{Co}-\mathrm{Fe}$ Magnetic Tunnel Junctions, J. Magn. Magn. Mater. 322, 996 (2010).

[34] R. J. Soulen, Jr, Measuring the Spin Polarization of a Metal with a Superconducting Point Contact, Science 282, 85 (1998).

[35] T. Kim and J.S. Moodera, Large Spin Polarization in Epitaxial and Polycrystalline Ni Films, Phys. Rev. B 69, 020403 (2004).

[36] F. Dalla Longa, J. Kohlhepp, W. de Jonge, and B. Koopmans, Influence of Photon Angular Momentum on Ultrafast Demagnetization in Nickel, Phys. Rev. B 75, 224431 (2007).

[37] M. Djordjevic, M. Lüttich, P. Moschkau, P. Guderian, T. Kampfrath, R. G. Ulbrich, M. Münzenberg, W. Felsch, and J.S. Moodera, Comprehensive View on Ultrafast Dynamics of Ferromagnetic Films, Phys. Status Solidi C 3, 1347 (2006).

[38] J. S. Moodera, J. Nassar, and G. Mathon, Spin-Tunneling in Ferromagnetic Junctions, Annu. Rev. Mater. Sci. 29, 381 (1999).

[39] S. X. Huang, T. Y. Chen, and C. L. Chien, Spin Polarization of Amorphous $\mathrm{CoFeB}$ Determined by Point-Contact Andreev Reflection, Appl. Phys. Lett. 92, 242509 (2008).

[40] J. Schmalhorst, S. Kämmerer, G. Reiss, and A. Hutten, Inelastic Electron Tunneling Spectroscopy and Bias Voltage Dependence of Magnetic Tunnel Junctions with Polycrystalline $\mathrm{Co}_{2} \mathrm{MnSi}$ Electrode, Appl. Phys. Lett. 86, 052501 (2005).

[41] S. Maat, M. J. Carey, and J.R. Childress, Current Perpendicular to the Plane Spin-Valves with CoFeGe Magnetic Layers, Appl. Phys. Lett. 93, 143505 (2008).

[42] M. J. Carey, S. Maat, S. Chandrashekariaih, J. A. Katine, W. Chen, B. York, and J. R. Childress, $\mathrm{Co}_{2} \mathrm{MnGe}$-Based CurrentPerpendicular-to-the-Plane Giant-Magnetoresistance SpinValve Sensors for Recording Head Applications, J. Appl. Phys. 109, 093912 (2011).

[43] D. Ebke, V. Drewello, M. Schaefers, G. Reiss, and A. Thomas, Tunneling Spectroscopy and Magnon Excitation in $\mathrm{Co}_{2} \mathrm{FeAl} / \mathrm{MgO} / \mathrm{Co}-\mathrm{Fe}$ Magnetic Tunnel Junctions, Appl. Phys. Lett. 95, 232510 (2009).
[44] P. Mavropoulos, I. Galanakis, V. Popescu, and P.H. Dederichs, The Influence of Spin-Orbit Coupling on the Band Gap of Heusler Alloys, J. Phys. Condens. Matter 16, S5759 (2004).

[45] A. Anguelouch, A. Gupta, G. Xiao, D. Abraham, Y. Ji, S. Ingvarsson, and C. Chien, Near-Complete Spin Polarization in Atomically-Smooth ChromiumDioxide Epitaxial Films Prepared Using a CVD Liquid Precursor, Phys. Rev. B 64, 180408(R) (2001).

[46] See Supplemental Material at http://link.aps.org/ supplemental/10.1103/PhysRevX.2.041008 for details in fabrication, additional data, and the derivation of the model.

[47] J. Walowski, G. Müller, M. Djordjevic, M. Münzenberg, M. Kläui, C.A.F. Vaz, and J.A.C. Bland, Energy Equilibration Processes of Electrons, Magnons, and Phonons at the Femtosecond Time Scale, Phys. Rev. Lett. 101, 237401 (2008).

[48] R. J. Elliott, Theory of the Effect of Spin-Orbit Coupling on Magnetic Resonance in Some Semiconductors, Phys. Rev. 96, 266 (1954).

[49] V. Kamberský, Spin-Orbital Gilbert Damping in Common Magnetic Metals, Phys. Rev. B 76, 134416 (2007).

[50] M. Fähnle, J. Seib, and C. Illg, Relating Gilbert Damping and Ultrafast Laser-Induced Demagnetization, Phys. Rev. B 82, 144405 (2010).

[51] K. Carva, M. Battiato, and P. Oppeneer, Ab Initio Investigation of the Elliott-Yafet Electron-Phonon Mechanism in Laser-Induced Ultrafast Demagnetization, Phys. Rev. Lett. 107, 207201 (2011).

[52] M. Djordjevic, M. Münzenberg, Connecting the Timescales in Picosecond Remagnetization Experiments, Phys. Rev. B 75, 012404 (2007).

[53] M.G. Münzenberg, Magnetization Dynamics: Ferromagnets Stirred Up, Nature Mater. 9, 184 (2010).

[54] H. Lee, Y. H. A. Wang, C. K. A. Mewes, W. H. Butler, T. Mewes, S. Maat, B. York, M. J. Carey, and J. R. Childress, Magnetization Relaxation and Structure of CoFeGe Alloys, Appl. Phys. Lett. 95, 082502 (2009).

[55] M. Zhu, B. D. Soe, R. D. McMichael, M. J. Carey, S. Maat, and J.R. Childress, Enhanced Magnetization Drift Velocity and Current Polarization in $(\mathrm{CoFe})_{1-x} \mathrm{Ge}_{x}$ Alloys, Appl. Phys. Lett. 98, 072510 (2011).

[56] P. V. Paluskar, J. J. Attema, G. A. de Wijs, S. Fiddy, E. Snoeck, J. T. Kohlhepp, H. J. M. Swagten, R. A. de Groot, and B. Koopmans, Spin Tunneling in Junctions with Disordered Ferromagnets, Phys. Rev. Lett. 100, 057205 (2008).

[57] S. Wurmehl, P. J. Jacobs, J. T. Kohlhepp, H. J. M. Swagten, B. Koopmans, S. Maat, M. J. Carey, and J. R. Childress, Local Formation of a Heusler Structure in CoFe-Al Alloys, Appl. Phys. Lett. 98, 012506 (2011).

[58] C. Utfeld, S. R. Giblin, J. W. Taylor, J. A. Duffy, C. ShentonTaylor, J. Laverock, S. B. Dugdale, M. Manno, C. Leighton, M. Itou et al., Bulk Spin Polarization of $\mathrm{Co}_{(1-x)} \mathrm{Fe}_{x} \mathrm{~S}_{2}$, Phys. Rev. Lett. 103, 226403 (2009).

[59] W.H. Butler, C. K. A. Mewes, C. Liu, and T. Xu, Rational Design of Half-Metallic Heterostructures, arXiv:1103.3855. 